\title{
Transcender les différends : une réaction possible aux situations de désaccord profond
}

The Appeal for Transcendence: A Possible Response to Cases of Deep

Disagreement

David Zarefsky

Traducteur : Sivan Cohen-Wiesenfeld

\section{(2) OpenEdition}

Journals

Édition électronique

URL : http://journals.openedition.org/aad/1251

DOI : 10.4000/aad. 1251

ISSN : 1565-8961

Éditeur

Université de Tel-Aviv

Référence électronique

David Zarefsky, «Transcender les différends : une réaction possible aux situations de désaccord profond ", Argumentation et Analyse du Discours [En ligne], 8| 2012, mis en ligne le 15 avril 2012,

consulté le 23 septembre 2019. URL : http://journals.openedition.org/aad/1251 ; DOI : 10.4000/aad. 1251

Ce document a été généré automatiquement le 23 septembre 2019.

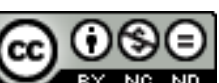

Argumentation \& analyse du discours est mis à disposition selon les termes de la licence Creative Commons Attribution - Pas d'Utilisation Commerciale - Pas de Modification 4.0 International. 


\title{
Transcender les différends : une réaction possible aux situations de désaccord profond
}

\author{
The Appeal for Transcendence: A Possible Response to Cases of Deep
}

Disagreement

David Zarefsky

Traduction : Sivan Cohen-Wiesenfeld

\section{La mise en vedette de l'accord}

1 Que le désaccord productif soit ancré dans un accord relève du truisme dans les théories de l'argumentation. Le partage d'un objectif commun, une même allégeance à des procédures spécifiques et l'adhésion à des vérités de base communes, sont considérés comme nécessaires pour que les argumentateurs puissent s'engager dans une confrontation digne de ce nom plutôt que de discuter sans s'entendre. Parmi les nombreux auteurs qui proposent une version de ce postulat, on peut citer Perelman et Olbrechts-Tyteca (1992: 87) : «le déroulement aussi bien que le point de départ de l'argumentation suppose l'accord de l'auditoire [...] d'un bout à l'autre, l'analyse de l'argumentation concerne ce qui est censé admis par les auditeurs ». Dans une veine similaire, Ehninger (1958: 28) écrit : «Le débat n'est pas un genre de conflit, mais de coopération. Les débattants [...] coopèrent au processus qui consiste à soumettre une proposition à un examen minutieux. [...] Ils souhaitent mettre leurs convictions à l'épreuve dans le respect du libre assentiment de l'autre ». Les croyances sur l'objectif de la procédure et ses modalités sont partagées par tous les partenaires du débat. Dans une étude visant à identifier les indicateurs de la situation d'argumentation, Brockriede (1975 : 182) y inclut « un cadre de référence partagé d'une manière optimale ». Pour lui, un débat entre deux personnes qui partagent trop de présupposés apparaît comme inutile, mais il devient impossible si elles n'en partagent pas suffisamment. Et MacIntyre (1984: 8) note l'impossibilité de raisonner à plusieurs lorsqu'il n'existe pas 
de normes partagées pour soutenir le discours rationnel. Il ne s'agit là que de quatre exemples représentatifs, parmi bien d'autres.

2 Il n'est guère difficile de comprendre pourquoi on s'accorde si bien sur la nécessité d'un accord. Tout d'abord, comme le notait Aristote, on ne débat pas de sujets sur lesquels on possède une certitude. Mais il faut bien que les arguments qui ne sont pas évidents en eux-mêmes soient évalués en référence à certaines normes si on veut mesurer leur degré de force et de pertinence. Deuxièmement, ni le fondamentalisme de la philosophie traditionnelle ni les standards universels de la logique formelle et des mathématiques ne permettent de traiter de l'argumentation ordinaire. Le consensus des argumentateurs sur les standards à appliquer devient dès lors le substitut de la validité formelle.

\section{Le désaccord profond}

Mais que se passe-t-il lorsque cette couche d'accord sous-jacente est absente, ou considérée comme manquante ? Tout argument avancé par l'un des locuteurs peut alors être remis en question par l'autre, dans un processus de régression potentiellement infini, parce qu'à nul instant l'interlocuteur n'est obligé d'accepter un argument en vertu de ses prises de positions antérieures. C'est Robert J. Fogelin (1985) qui a le premier défini cet état de chose par l'expression de désaccord profond (deep disagreement). Dans ce cas de figure, tous les arguments du locuteur sont fondés sur des présuppositions que l'autre rejette. Le désaccord profond constitue ainsi une situation limite dans laquelle l'argumentation devient impossible. La plupart des débats qui s'y réfèrent supposent qu'il s'agit d'un cas relativement rare, qui ne contrevient pas à l'utilité de l'argumentation envisagée comme le moyen à l'aide duquel les locuteurs ordinaires résolvent pacifiquement leurs différends. Dans la mesure où de nombreuses discussions portant sur l'argumentation la situent dans un cadre dialogal, elles écartent d'emblée la question du désaccord profond, comme si elle était sans conséquence audelà de ses effets immédiats sur les participants de l'échange.

Or, ces hypothèses sont toutes deux sujettes à caution: la première en raison de la montée du fondamentalisme, et la seconde parce que le désaccord profond s'est avéré politiquement utile. La génération passée a été témoin de l'ascension du fondamentalisme dans le cadre des principales traditions religieuses : judaïsme ultraorthodoxe, chrétiens évangélistes et islam radical. Le fondamentalisme rejette l'hypothèse moderne de la faillibilité humaine, et la tolérance envers des points de vue diversifiés qui en découle. Les fondamentalistes croient en effet qu'il est possible de connaître la volonté de Dieu avec certitude. Dieu l'a rendue explicite, et la Parole divine peut être lue et comprise par quiconque est désireux de le faire. S'écarter de cette parole afin de faire preuve de tolérance envers ceux qui sont plongés dans l'erreur n'est pas seulement inutile, mais pervers ; c'est une démarche qui fait endosser aux justes les péchés des hommes sans foi.

5 En raison du conflit entre le fondamentalisme et le modernisme (ou même plus, le postmodernisme), de nombreux désaccords sont interprétés par les uns en termes moraux et religieux, et par les autres, en termes pragmatiques et laïques. Cela est valable non seulement pour les questions d'identité et de droits individuels comme l'avortement, le féminisme et les droits des homosexuels, mais aussi, et de plus en plus, pour des sujets allant des impôts et de la politique fiscale à la protection de l'environnement, en 
passant par les théories de la justice criminelle et pénale. Même coupés de toute dimension religieuse explicite, les débats publics sur la santé, les facteurs de croissance économique et les réglementations financières semblent de plus en plus fréquemment glisser vers des affirmations de principe sur les droits de l'individu et le rôle de l'Etat, principes sur lesquels l'accord semble impossible. Ainsi, les défenseurs des deux parties s'adressent de plus en plus à ceux qui pensent comme eux: dès lors, l'idée que l'argumentation peut être utilisée productivement pour résoudre les différends se vide de son sens. La difficulté est peut être plus prononcée aux Etats-Unis en raison de la grande influence qu'y exerce le fondamentalisme. D'après ce que j'ai pu lire sur le problème de l'immigration, de l'intégration économique de l'Union européenne et de la question de savoir si la religion doit tenir un rôle public, il semble cependant que l'Europe connaisse une évolution similaire.

6 La seconde hypothèse est également discutable. Dans la mesure où le désaccord profond est utile sur le plan politique, il peut affecter tous ceux qui sont intéressés au programme politique en jeu. C'est ce qui s'est passé aux Etats-Unis, particulièrement ces vingt dernières années. Le parti minoritaire a souvent vu plus d'avantages à s'opposer purement et simplement à l'administration au pouvoir, qu'à travailler en coopération avec elle pour résoudre les problèmes. Il s'est comporté comme si les deux parties étaient dans une situation de désaccord profond, menant ainsi le débat public à une impasse. Les problèmes qui se posent restent sans solution, ou doivent être résolus au moyen de chiffres, d'argent ou encore par la force, plutôt que par le discours raisonné.

7 Cette tendance s'est encore accentuée depuis l'élection de Barak Obama. Les Républicains au Sénat et à la Chambre des Représentants ont presque unanimement voté contre la plupart des initiatives du Président, les retardant ou leur faisant obstruction, et obligeant Obama à des marchés (des " deals ») politiques à l'ancienne pour assurer la cohésion du parti démocrate. Il ne s'agit peut-être pas d'un véritable cas de désaccord profond, bien qu'il ait été traité comme tel. Lorsque Obama incorpora à ses projets de loi des propositions que les Républicains avaient précédemment soutenues, ceux-ci changèrent de position et votèrent contre. Ils ont dépeint ses orientations centre-gauche comme relevant du "socialisme ", et considéré le débat comme une lutte entre l'extension du pouvoir gouvernemental et la protection de la liberté du peuple, c'est-à-dire comme le choc de deux visions du monde incompatibles. L'administration Obama n'a certes pas été la seule à faire l'objet d'une telle division partisane, mais le phénomène semble avoir été plus important et systématique que sous George W. Bush ou Bill Clinton.

8 Si donc le désaccord profond représente un phénomène répandu et important, les études d'argumentation se doivent de lui accorder une attention plus soutenue. Il y a près de dix ans, ces questions ont été explorées en profondeur par Nola Heidlebaugh (2001: xi). Le problème qu'elle posait était: "Sans consensus sur les normes de la raison, comment pouvons-nous bénéficier d'un bon débat public? Et sans l'éloquence et la richesse d'un tel débat, comment pouvons-nous raisonner ensemble pour parvenir à un consensus sur les problèmes qui se posent à nous ? "Ces questions constituent pour les chercheurs en argumentation autant de motifs pour explorer les moyens de surmonter les désaccords profonds et de ramener le débat public sur une piste productive. 


\section{L'incommensurabilité : le début de l'analyse ou sa fin?}

Heidlebaugh observe qu'en cas de désaccord profond, les positions en concurrence sont incommensurables. Elles ne peuvent être comparées, car les procédures pour établir et légitimer les jugements ne sont pas fondées sur les mêmes règles. Mais, selon Heidlebaugh, si ce phénomène d'incommensurabilité rend toute poursuite de la discussion impossible pour le logicien, pour le rhétoricien au contraire le plaisir ne fait que commencer. L'un des argumentateurs, ou plusieurs d'entre eux, doivent trouver le moyen de sortir de l'impasse et de poursuivre le débat sur d'autres bases. Comme l'écrit Heidlebaugh (2001: 74), «il incombe au rhéteur de trouver quelque chose à dire qui contribue à résoudre le problème particulier qu'il a détecté ». L'incommensurabilité n'est pas un état qui nécessite des "soins", c'est une situation qui en appelle à la sagesse pratique. La tâche de l'argumentateur est de faire émerger « une position particulière à partir de laquelle émergent de nouveaux points de différence et de similitude » car ce faisant, il «met en évidence l'importance de découvrir de nouvelles choses à dire " (2001 : 128). Bien que Heidlebaugh étudie la tradition de la rhétorique classique et affirme que les lieux communs, les topoi et la stasis sont les ressources de "l'invention", elle n'identifie pas de stratégies particulières pour transcender l'incommensurabilité. C'est ce que voudrais faire à présent, au moyen d'une réflexion fondée sur l'expérience et l'analyse de cas spécifiques.

\section{Stratégies pour surmonter les désaccords profonds}

10 Je regroupe les stratégies possibles par paires, sous les rubriques de mise en contradiction de l'interlocuteur, d'englobement, de temps et de changement de terrain. Chacune d'entre elles reflète l'hypothèse selon laquelle avancer des arguments d'une manière habituelle serait inefficace pour sortir de l'impasse, car incompatible avec le point de vue de l'autre. Il faut repenser différemment le choc des opinions conflictuelles.

\subsection{Mise en contradiction de l'interlocuteur : hypocrisie et ad hominem circonstanciel}

11 Les deux premières stratégies tentent de pénétrer dans le cadre de référence de l'adversaire en le discréditant pour motif d'incohérence. Elles reposent sur la loi de non-contradiction selon laquelle un argument solide ne peut se contredire lui-même.

12 L'accusation d'hypocrisie consiste à prétendre que le locuteur soutient une position qui n'est pas cohérente avec un point de vue qu'il ou elle a précédemment avancé. En l'absence d'explication de ce changement, on conclut logiquement que le locuteur est hypocrite et agit par opportunisme, et non selon des principes.

$13 \mathrm{Au}$ début de l'année 2010, certains dirigeants du parti républicain aux Etats-Unis s'opposèrent à une augmentation du financement par l'Etat pour relancer l'économie afin de ne pas creuser un déficit budgétaire déjà important en gonflant la dette publique. Cependant, beaucoup de ces mêmes parlementaires avaient voté en faveur de déficits budgétaires encore plus élevés sous l'administration Bush, pour financer le coût 
de la guerre en Irak et l'indemnisation des médicaments sur ordonnance pour les personnes âgées, ou pour parer aux diminutions d'impôts non accompagnées de la nécessaire réduction des dépenses. Un Démocrate pourrait répondre aux plaintes des Républicains au sujet du déficit budgétaire de la manière suivante :

1. Le déficit vous dérange maintenant.

2. Mais il ne vous dérangeait pas quand votre parti était au pouvoir.

3. [votre changement de position n'a pas d'explication apparente]

4. Vous êtes par conséquent un hypocrite. Vous ne vous souciez pas du déficit mais cherchez uniquement un bénéfice politique. Tout ce que vous recherchez est de vous différencier des partisans du Tea Party ${ }^{1}$ et de consolider votre base politique.

5. Par conséquent, votre argumentation ne repose sur aucun fondement de principe et doit être rejetée.

6. Puisque votre point de vue ne peut passer le test de la cohérence, et que vos normes sont en conflit avec les miennes, c'est ma position qui l'emporte sur la vôtre par élimination.

Ces étapes ne seront pas formulées explicitement, mais ce sont bien celles qui soustendent cette démarche. Je promeus mon point de vue non en l'étayant pas des raisons supplémentaires, mais en démontrant que le vôtre ne résiste pas à l'épreuve de cohérence.

Bien sur, cette stratégie est vulnérable. Elle dépend de l'hypothèse non formulée selon laquelle le changement de position de l'opposant ne repose sur aucune justification apparente. Personne ne présente sciemment des positions incohérentes susceptibles de l'exposer à une accusation d'hypocrisie, si bien que l'opposant déploiera des efforts considérables pour établir une différence entre les différentes positions mentionnées par son contradicteur. Il se peut que le déficit budgétaire soit justifié lorsqu'il y va de la sécurité nationale, mais pas pour relancer l'économie. Ou bien qu'il soit valable lorsqu'il permet de placer plus d'argent entre les mains des individus, mais pas lorsqu'il implique des dépenses excessives de la part du gouvernement. Ou encore qu'il est éventuellement admissible s'il est effectué au profit des personnes âgées, mais pas quand il s'agit de l'ensemble de la population. Chacun de ces arguments nécessiterait bien sûr d'être étayé, mais la charge de la preuve serait aisée précisément parce que nous supposons que les locuteurs n'avancent généralement pas des arguments hypocrites.

L'argument ad hominem circonstanciel est lié à l'accusation d'hypocrisie. Il ne s'agit pas d'une attaque contre la personne de l'adversaire, mais plutôt de l'affirmation selon laquelle ses positions sont en contradiction avec son comportement dans une situation spécifique. Du lieu commun selon lequel « les actes sont plus probants que les paroles », on infère que les actions d'une personne révèlent ses véritables engagements bien plus que ses discours (Walton $1998: 2-6,108-112$ ). Le point de vue est donc disqualifié parce qu'il ne peut s'appuyer sur les propres actes de l'argumentateur. Et puisque mon point de vue représente l'alternative du vôtre, c'est lui qui l'emporte, une fois de plus par défaut. Johnstone (1959) a été jusqu'à suggérer que toute argumentation philosophique valide était de cet ordre.

Supposons que A soit un avocat pour qui la protection des libertés civiles est une valeur primordiale. A a ouvertement pris position sous l'administration Bush contre les efforts de conférer plus de pouvoirs au Président pour lutter contre le terrorisme, dans l'idée que de telles mesures violaient le droit individuel à la vie privée. Pourtant, A accepte une invitation à plaider devant la Cour Suprême pour défendre l'extension de ces 
mêmes pouvoirs lorsque l'administration Obama cherche à les conserver. "Vous ne devez pas être vraiment attaché aux libertés civiles", allègue alors un critique, «puisque vous abandonnez cet engagement pour bénéficier de l'occasion de défendre le président Obama devant la Cour Suprême ". Les actes de A révèlent son véritable engagement - envers l'administration Obama - et discréditent l'attachement aux libertés civiles qu'il professe. Cette dernière position disqualifiée, le point de vue alternatif prévaut par élimination: A pense que la défense de la nation contre les terroristes l'emporte sur la protection des libertés civiles, au moins dans le cas présent - hiérarchie que son interlocuteur tente de discréditer.

Comme dans l'exemple de l'hypocrisie, la réponse probable de l'opposant sera d'établir une nette différence entre les deux situations, en plaçant les paroles et les actes sur deux plans différents. Il ou elle pourrait s'opposer à de nouvelles restrictions aux libertés civiles, tout en soutenant que la suppression des limitations existantes serait susceptible de donner aux autres nations l'impression que les Etats-Unis sont faibles. Ou bien il pourrait vouloir conserver les restrictions actuelles parce qu'il a confiance dans le fait qu'Obama en fera un usage judicieux et n'y aura recours qu'en dernier ressort, confiance qu'il n'avait pas dans le Président Bush. Si l'adversaire parvient à établir la distinction entre la situation dans laquelle A a énoncé ses engagements et celle à propos de laquelle il a été mis sur la sellette, l'ad hominem circonstanciel censé permettre de surmonter le désaccord profond échoue, et la perception que celui-ci prévaut subsiste. Alternativement, l'opposant pourrait prétendre qu'il ou elle fait juste son métier d'avocat, veillant à ce que chaque client bénéficie de la meilleure défense possible.

\subsection{Stratégie d'englobement : incorporation et subsomption ${ }^{2}$}

19 La seconde paire de stratégies concerne la façon d'englober des arguments dans un même ensemble. L'une est l'incorporation, stratégie dans le cadre de laquelle le locuteur fait entrer les arguments incommensurables (et les propositions qui les accompagnent) dans un ensemble plus large. Le succès de cette stratégie dépend de la mesure dans laquelle les deux interlocuteurs perçoivent la situation d'impasse comme intolérable. Aucun des deux n'est prêt à faire de concessions, mais ils ne sont pas non plus disposés à perpétuer une situation bloquée. L'administration Obama a tenté d'adopter cette approche lors de la préparation du projet de loi sur le financement des soins médicaux, en y incorporant certaines propositions républicaines, comme la " réforme sur la responsabilité pour négligence » (tort reform) visant à réduire les poursuites judiciaires pour faute professionnelle. Les partisans d'obama n'ont pas renoncé à leur propre point de vue concernant les causes des coûts des soins de santé ils ont même soutenu que la «réforme sur la responsabilité pour négligence » ne résoudrait qu'une très petite partie du problème - mais ils ont jusqu'à un certain point inclus cette réforme dans leur projet de loi pour que les Républicains puissent soutenir la réforme de la santé conformément aux principes qu'ils professent.

Cet effort a clairement échoué, témoignant ainsi des difficultés que présente la stratégie d'incorporation. Il faut en effet que les deux interlocuteurs souhaitent sortir de l'impasse. Dans le cas présent, l'adoption de la loi sur la réforme de la santé, si elle ne se faisait pas dans leurs propres termes, ne constituait pas une priorité pour les opposants républicains. Même si la réforme de la responsabilité faisait partie du projet 
de loi, elle ne suffisait pas à leur faire avaler d'autres éléments du projet qu'ils trouvaient choquants. Certains ont effectivement préféré voter contre le projet de loi alors que d'autres, voyant que le gouvernement souhaitait désespérément arriver à faire passer un quelconque projet de loi, ont préféré tenir bon pour voir si une position dure entraînerait davantage de concessions.

Liée à l'incorporation, la subsomption est une stratégie qui cherche à subsumer les deux points de vue inconciliables à l'intérieur d'un cadre plus large. L'un des interlocuteurs entreprend la démarche, et invite l'autre à coopérer. La forme standard de l'argument serait quelque chose comme

1. Nos positions $X$ et $Y$ paraissent incommensurables.

2. Si vous soutenez $X$, vous devez soutenir $Z$ parce que $Z$ fera avancer la cause de $X$.

3. Si je soutiens $Y$, je dois soutenir $Z$ parce que $Z$ fera progresser la cause de $Y$.

4. Nous pouvons donc subsumer notre désaccord sur $X$ et $Y$ sous notre accord sur $Z$.

La différence entre l'incorporation et la subsomption est que la première a uniquement pour but de sortir d'une impasse argumentative, alors que la seconde vise également à développer un phénomène d'identification positive avec le facteur commun $\mathrm{Z}$.

La controverse sur l'avortement offre un exemple intéressant de tentative de subsomption. Le débat entre "partisans de la vie» (pro-life) et ceux «du choix» ou liberté de choisir l'avortement (pro-choice) débouche rapidement sur une impasse; les points de vue en concurrence reflètent des visions du monde incommensurables sur des questions fondamentales comme celle de savoir si notre propre corps nous appartient. Mais les argumentateurs peuvent tenter de subsumer ces différences sous la question de savoir quelle est la meilleure manière d'empêcher les grossesses involontaires. Les deux parties sont intéressées à ce problème, dont la résolution réduirait les cas dans lesquels se pose le dilemme moral de l'avortement. Sur le plan pratique, cette démarche pourrait être productive.

Mais là encore, l'expression «sur un plan pratique » est un signal d'avertissement. Le conflit entre "partisans de la vie» et "partisans du choix» ne se situe pas sur un terrain pratique, mais au niveau des principes. On peut imaginer le conflit fonctionnant exactement de la même manière sans aucun rapport avec le fait que les deux parties soutiennent ou non un programme commun pour réduire les grossesses involontaires. Chaque partie pourrait très bien accepter la nécessité de réduire les grossesses non programmées, en retirer les bénéfices, et retourner immédiatement à des points de vue enracinés dans des principes et des visions du monde incommensurables.

Incorporation et subsomption peuvent être combinés. Un exemple célèbre en est le débat au Sénat américain sur le Compromis de 1850, présenté à l'origine comme un projet de loi omnibus ${ }^{3}$ destiné à résoudre tous les conflits en suspens sur l'esclavage. Plusieurs objectifs incompatibles furent plus ou moins incorporés dans un même ensemble, mais ces actions individuelles furent subsumées sous la rubrique d'une même finalité. Les deux parties souhaitaient la résolution définitive de la controverse sur chaque centimètre carré du territoire américain. Dans leurs plateformes pour les élections de 1852, les deux partis politiques s'engagèrent à respecter le compromis de 1850 comme solution finale de la controverse ${ }^{4}$. Le compromis était pourtant fragile. Avec le temps, chaque partie pouvait penser (et c'est se qui arriva finalement) qu'elle était victime d'une injustice, et donnait plus qu'elle ne gagnait. C'est plus ou moins ce qui s'est passé pendant les années qui ont précédé la Guerre civile américaine. 


\subsection{La stratégie du temps : lassitude et urgence} de l'impasse argumentative. L'une d'entre elle est le recours à la lassitude. Les cas de désaccord profonds peuvent demeurer dans l'impasse pendant un certain temps. Une des parties peut finalement décider que la durée de la controverse est devenue disproportionnée à son importance et tenter de convaincre l'autre de dépasser cette situation. Il se peut même que les adversaires aient quitté la scène, et que leurs successeurs soient moins disposés à poursuivre le combat. Ou bien la controverse peut se trouver dépassée du fait que ses conséquences sur le statut des participants ne sont plus aussi importantes. Ou encore l'impasse en elle-même peut être devenue gênante parce que « la vie est trop courte " pour rester obstinément fixé sur une seule et même question. Pour l'une ou l'autre de ces raisons, l'une des parties peut tenter de convaincre l'autre que le moment est venu, non pas nécessairement de résoudre le désaccord profond, mais au moins de le mettre de côté et de passer outre.

C'est une attitude de ce type qui a motivé le défunt Premier Ministre Yitzhak Rabin à faire des ouvertures auprès des Palestiniens pour entamer des négociations de paix au début des années 1990. De longues années d'hostilité amère avaient prélevé un prix terrible. Les Palestiniens n'étaient pas devenus les amis d'Israël, mais comme Rabin l'avait noté avec justesse, on n'a pas besoin de faire la paix avec ses amis.

Comme certaines des autres stratégies, le piège de celle-ci est qu'elle dépend d'un état mutuel de lassitude. La partie qui avance cet argument doit convaincre l'autre de ressentir la même chose, sinon, cette dernière peut considérer l'appel à la lassitude comme un aveu de faiblesse. Si la partie «non lasse» tient bon, l'autre peut perdre courage et renoncer au combat. C'est plus ou moins ce qui s'est passé dans le cas de la guerre du Vietnam.

Cependant, plus souvent qu'à la lassitude, les locuteurs auront recours à l'urgence causée par une crise pour dépasser un désaccord profond. Le sous-entendu est que le désaccord profond est un luxe qui peut être toléré en temps normal, mais pas actuellement ; l'heure est à l'essentiel et la gravité de la situation exige une prompte réaction.

30 A l'automne 2008, le système financier américain était menacé d'une implosion avec des risques probables de répercussion dans le monde entier. Pour éviter le désastre, l'administration Bush préconisa des infusions massives d'argent liquide et de garanties bancaires afin de restaurer la confiance dans l'économie américaine. Ses propositions de "sauvetage de dernière minute » furent critiquées par de nombreux membres du parti de Bush lui-même, convaincus des capacités de résistance du marché lorsqu'il est laissé à lui-même, sans intervention. Même le Président Bush admit qu'il était gêné par les mesures qu'il proposait et qu'en temps normal, il ne les aurait pas suggérées. Mais la perspective de la menace d'une crise majeure l'obligeait à laisser de côté son engagement idéologique. Ce qui ne fut pas le cas pour de nombreux Républicains de la Chambre. Peu disposés à accepter l'idée que les Etats-Unis faisaient face à un effondrement financier, ils firent en un premier temps échouer les mesures appelant à des subventions d'urgence (bailout). Ce n'est que lorsque la Bourse, en réaction, s'écroula, qu'ils reconsidérèrent leur position et firent passer une version modifiée du projet d'urgence.

Argumentation et Analyse du Discours, 8 | 2012 
31 La reconnaissance d'un état de crise dépend du regard de l'observateur. Si l'une des parties tient bon et refuse de définir la situation en termes de crise, cet argument sera inefficace, voire contre-productif. D'un autre côté, la perception d'une situation de crise est un levier d'action puissant. C'est peut-être la raison pour laquelle le chef du Personnel de la Maison Blanche, Rahm Emanuel a conseillé : «Never let a crisis go to waste » ( «Il ne faut jamais gaspiller une crise »).

\subsection{Le changement de terrain : emprunt « inter-champs » et recadrage}

La dernière paire de stratégies est peut-être la plus ambitieuse en ceci qu'elle consiste à déplacer le terrain sur lequel se manifeste le désaccord profond. Cette stratégie est ce que Willard (1983:267-270) a appelé un emprunt inter-champs. Williard observe que les champs d'argumentation possèdent leurs normes distinctes de preuve et de raisonnement, mais également que de nombreux conflits ne peuvent être rattachés uniquement à un champ unique. L'euthanasie, par exemple, est à la fois un problème scientifique et moral, mais scientifiques et moralistes envisageront probablement la question différemment, ce qui pourra aboutir à un désaccord profond, sauf si l'une des parties accepte, dans l'intérêt de l'argumentation, d'invoquer les normes du champ de l'adversaire pour le vaincre sur son propre terrain. Pour rendre compte des origines de l'homme, par exemple, les moralistes pourraient «emprunter » la théorie scientifique de l'évolution et tenter de démontrer sur une base scientifique qu'elle est infondée. Ou, inversement, le scientifique peut emprunter la figure du moraliste pour prétendre qu'une explication biblique de la création n'est pas en contradiction avec les jugements concernant l'évolution.

L'intérêt de «l'emprunt" à un autre champ est de mettre les deux côtés de l'argumentation sur le même plan, puis de discréditer le champ de l'autre dans ses propres termes. Mais l'emprunteur ne sera jamais aussi bien documenté que la personne qui occupe véritablement le champ dont l'argumentateur choisit de se réclamer. La deuxième partie peut trouver des raisons prouvant que l'emprunt n'est ni sincère ni juste, ou alléguer que l'emprunteur possède des notions stéréotypées et limitées du champ dans lequel il prétend se situer.

La seconde stratégie liée au changement du terrain argumentatif est le recadrage, par lequel une partie cherche à faire passer le conflit d'un contexte ou cadre de référence à un autre. Les célèbres débats Lincoln-Douglas de 1858 en offre un exemple intéressant. La question centrale était de savoir si l'esclavage devait ou non être étendu aux nouveaux territoires ${ }^{5}$. Lincoln pensait que non, parce que l'esclavage était mauvais en lui-même et qu'il n'était pas logique de vouloir étendre quelque chose de mauvais. Il appuyait son point de vue sur une argumentation morale de fond (Zarefsky 1990). Mais pour Douglas, la vraie question était de savoir qui devait décider si l'esclavage était bon ou mauvais. Il s'agissait pour lui d'une question morale complexe sur laquelle des personnes de bonne foi étaient en désaccord, et il ne se permettait pas de prendre de décision pour ceux qui allaient véritablement vivre dans ces territoires et en supporter les conséquences. Il se fit donc le champion de la "souveraineté populaire», en appuyant son point sur une argumentation procédurale. Ces positions, l'une de fond, l'autre de procédure, étaient incommensurables. C'est peut-être la raison pour laquelle les arguments sur la moralité de l'extension de l'esclavage occupèrent une si petite 
partie du temps de débat. Au lieu de cela, les deux candidats débattirent, entre autres choses, de ce que les fondateurs de la nation auraient fait sur le sujet s'ils avaient été vivants. Les candidats ont donc déplacé le débat d'un cadre moral à un cadre historique. Ici, il pouvait y avoir des normes partagées, parce que les deux hommes vénéraient les pères fondateurs, et tous deux pensaient que leur perspicacité pouvait éclairer les délibérations actuelles. Et sur ce point, il pouvait y avoir débat, parce que la question ne pouvait pas être résolue de manière décisive. En effet, les fondateurs n'avaient jamais été confrontés au problème de près, et il fallait donc déduire de leurs déclarations, ou des actes accomplis à propos d'autres questions au fil des ans, la position qu'ils auraient vraisemblablement adoptée.

Le recadrage était utile aux débats Lincoln-Douglas car les deux candidats pouvaient accepter le cadre de substitution, chacun pensant qu'il fonctionnerait à son avantage. Mais ce n'est pas toujours le cas. Le locuteur qui cherche à déplacer le cadre de référence peut se heurter à des résistances. Par exemple, Lincoln ou Douglas auraient pu insister sur le fait que cette spéculation historique constituait un détournement non pertinent des problèmes du moment. Ou bien les candidats auraient pu se trouver en désaccord profond sur le choix des preuves historiques pertinentes, ou sur leur interprétation correcte.

\section{Deux études de cas}

Il faut remarquer que chacune de ces huit stratégies pour dépasser les désaccords profonds représente une option valable dotée d'une force probante, mais qu'aucune d'entre elles n'est assurée du succès. Comme toute stratégie rhétorique, elles doivent être adaptées à une situation particulière. Dans certains cas, un locuteur pourra montrer qu'elles conviennent parfaitement, tandis que dans d'autres, un argumentateur différent parviendra à démontrer qu'elles sont inapplicables. Ce point sera éclairé par deux études de cas dont l'une a été couronnée de succès alors que l'autre s'est soldée par un échec.

\subsection{Johnson sur l'éducation}

37 Aux Etats-Unis, l'éducation élémentaire et secondaire a traditionnellement été considérée comme relevant de la responsabilité de l'Etat et des collectivités locales en même temps que du secteur privé. Bien qu'il y ait eu des exceptions, comme les subventions fédérales octroyées aux écoles situées à proximité des bases militaires qui contribuent à l'enrôlement des jeunes gens dans l'armée, l'aide fédérale générale à l'éducation n'a pas fait partie de la politique gouvernementale jusque dans les années 1960, même si une majorité de législateurs et de la population se déclarait en sa faveur. La raison en résidait partiellement dans le fait que les partisans de cette mesure étaient divisés entre eux sur la question de savoir si l'aide fédérale devait être étendue aux écoles confessionnelles. Pour certains, cela aurait signifié l'abolition de la séparation entre l'Eglise et l'Etat, créant une institutionnalisation de la religion en violation de la Constitution des Etats-Unis. Si le projet de loi sur l'éducation comportait un tel article, et bien qu'ils se déclarent en faveur du principe de l'aide fédérale à l'éducation, ils s'y seraient opposés. Mais laisser tout simplement les écoles religieuses en dehors du projet de loi n'était pas non plus une solution; d'autres législateurs étaient convaincus 
qu'un tel comportement serait discriminatoire par son refus d'offrir une protection légale aux familles qui souhaitaient donner à leurs enfants une éducation religieuse. L'argent de leurs impôts aurait servi au développement de l'éducation, mais ils n'auraient pu eux-mêmes en bénéficier. Ceci, disaient certains législateurs, constituerait une ingérence dans le libre exercice de la religion, également en violation de la Constitution américaine. Pendant ce temps, la minorité opposée à l'aide fédérale à l'éducation en toute circonstance n'avait pratiquement pas besoin de défendre son point de vue puisque les partisans de l'aide fédérale étaient en désaccord profond entre eux sur une question subsidiaire.

Ces questions restèrent donc dans une impasse jusqu'à l'accession à la présidence des Etats-Unis de Lyndon Johnson. Ce dernier entrepris un recadrage avec succès. Il insista pour que le sujet ne soit pas considéré comme une aide à l'école laïque ou religieuse, mais comme une aide aux enfants (Dallek 1998: 197). Sa proposition comprenait des formules d'aide fondées sur le nombre d'enfants dans une juridiction relatives aux familles dont les revenus étaient en-dessous du seuil de pauvreté. En théorie, les enfants devaient utiliser cette aide pour faire leurs études dans n'importe quelle école. En pratique, les écoles agissaient comme des agents pour les enfants, demandant une aide basée sur le nombre d'enfants concernés. Cette reformulation du problème, déplaçant le cadre, satisfaisait les deux groupes de partisans qui se trouvaient auparavant dans une impasse. Les deux parties pouvaient considérer la proposition ainsi reformulée comme étant en harmonie avec leurs convictions profondes.

\subsection{Zarefsky sur l'avortement}

39 Ma seconde étude de cas a un résultat moins positif, surtout dans la mesure où j'y suis moi-même impliqué. Il y a quelques années, j'ai produit un cours vidéo sur l'argumentation destiné à la vente (Zarefsky 2005). Dans l'un des premiers cours, j'avais avancé que l'argumentation supposait l'incertitude puisqu'il n'est pas nécessaire de débattre de sujets sur lesquels nous possédons des certitudes. L'un de mes exemples était qu'il n'y avait pas moyen de savoir avec certitude quand commence la vie humaine; et j'ajoutais que c'est l'une des raisons majeures pour lesquelles la controverse sur l'avortement était tellement difficile à résoudre.

Un peu plus tard, je reçus de plusieurs adolescents lycéens du Minnesota un groupe de lettres pratiquement identiques les unes aux autres. Elles s'offusquaient gravement de l'affirmation qu'il n'existait aucun moyen de savoir quand commençait la vie humaine. Bien sûr que si, répondaient-ils. Tout le monde sait que la vie humaine commence à la conception; c'est écrit dans la Bible. Ils citaient ce qu'ils pensaient être les versets bibliques applicables. L'avortement est donc un meurtre, me disaient-ils. Certaines personnes pensent apparemment que la société et le gouvernement peuvent tolérer le meurtre d'un enfant à naître. C'est pour cela qu'il y a controverse.

J'aurais pu ignorer ces lettres, mais je voulais rendre hommage à leur ton sérieux et respectueux. J'ai donc répondu à ces étudiants. Je tentai un emprunt «inter-champs ", en l'occurrence l'utilisation de leur source privilégiée, la Bible, pour alléguer que l'origine de la vie humaine était incertaine. Je citai des passages du livre de l'Exode disant que si un homme frappait une femme enceinte et que celle-ci mourrait, il serait puni de meurtre. Si la femme restait vivante mais avortait, la peine était moins lourde et limitée à des indemnités financières. Le fœtus était donc moins valorisé qu'une 
personne vivante. Voilà donc une preuve, disais-je, qui remettait en question leur opinion selon laquelle la Bible considérait l'avortement comme un meurtre. Mon but, je le rappelle, n'était pas de réfuter catégoriquement leur argument mais uniquement de montrer que son statut était incertain, parce que le point où la vie humaine (à la différence de l'animal) commençait était incertain. Il me semblait que la charge de la preuve à apporter était faible, et que j'y avais fait face.

42 Je fus surpris de recevoir une réponse, non pas des étudiants, mais de leur professeur. Celle-ci me remerciait d'avoir répondu aux étudiants mais se plaignait de ce que je les induise en erreur. Sa traduction du texte de l'Exode faisait la distinction entre l'expulsion d'un fœtus vivant et la mort d'un fœtus dans l'utérus. Elle disait que dans un cas on appliquait des sanctions financières, alors que dans l'autre, la peine capitale était assurée. Puisque ma traduction ne faisait pas cette distinction, disait-elle, elle était erronée sinon frauduleuse, et pour ma propre édification, je devais me procurer un meilleur texte et abjurer mon hérésie. Elle priait pour mon âme (je note en passant qu'elle ne demandait pas quel était mon texte et ne semblait pas s'en soucier).

43 Je ne suis pas un théologien érudit, mais je pense que le problème ici est que le verbe hébreu d'origine est ambigu quant à la question de savoir si le fotus est expulsé vivant ou mort. J'ai quelques raisons de penser que ma traduction était plus fiable que la sienne, puisqu'elle reflète des conventions en usage à l'époque où le texte biblique fut rédigé. Mais tout ce que j'essayais d'établir était que le sujet était incertain et constituait donc un objet d'argumentation nécessaire et approprié.

44 A ce stade, j'abandonnai la discussion. L'attaque de ma correspondante sur ma source sans même savoir ce qu'elle était me suggérait que sa conception du monde ne supporterait pas l'incertitude. Toute contre-épreuve serait rejetée à l'avance et donc le débat était d'ores et déjà clos. C'était un cas d'opposition entre fondamentalisme et modernisme. Ma position dépendait fondamentalement de la notion d'incertitude ; la sienne était basée sur la certitude ; et le fossé semblait impossible à combler. Mon effort d'emprunt inter-champs fut un échec parce que de son point de vue, je n'avais pas réussi à établir ma bonne foi à l'intérieur de son champ.

Ceci étant, je n'ai peut-être pas fait ce qu'il fallait. J'aurais peut-être dû déployer plus d'efforts, soit en défendant le choix de mon texte, soit en essayant de trouver un passage dans sa propre traduction qui fonctionne contre son argumentation, ou peutêtre même en cherchant un terrain autre que l'autorité de la Bible. Mais j'ai pensé que de tels efforts seraient futiles, j'avais autre chose à faire, et j'ai donc laissé la discussion au point où les interlocuteurs s'accordaient à ne pas être d'accord. Je ne modifierais pas l'affirmation où je disais dans ma conférence que le moment où débute la vie humaine était incertain, et elle n'abandonnerait pas sa conviction sur l'inexactitude de cette affirmation. Rester dans l'impasse est un résultat sans grand dommage dans un dialogue interpersonnel entre deux individus. Pourtant, comme je l'ai suggéré cidessus, la chose est moins inoffensive quand elle se démultiplie et affecte la politique sociale autant que le jugement individuel.

\section{Conclusion}

Dans les modèles d'argumentation dialogique, les résultats n'affectent généralement que les partenaires individuels. Dans les modèles d'argumentation rhétorique, cependant, il existe un tiers, un auditoire, qui est affecté par l'échange. Comme Schmitt 
(2010: 10) l'a récemment écrit, « les conséquences de cette rhétorique apocalyptique et de cette politique de tout ou rien retombe sur nous tous lorsque le gouvernement est dans l'incapacité d'agir \%. Un désaccord profond perpétuel n'est guère propice au public. La demande de faire avancer la discussion peut exercer une pression extérieure sur les adversaires et les pousser à sortir de l'impasse. Actuellement aux Etats-Unis, le mécontentement du public face au blocage du débat politique se répand de plus en plus. Mais il se manifeste d'une manière peu sophistiquée, et, à mon avis, inefficace : sous la forme d'un mouvement populiste de droite sans discernement symbolisé par le Tea Party et son exigence de "récupérer le gouvernement ». Il a causé un préjudice notable aux fonctionnaires en place et développé un discours politique qui exalte le manque d'expérience en l'érigeant en vertu. Ce préjudice populaire entrave les efforts de collaboration pour trouver des compromis, sous prétexte que cela signifierait pactiser avec l'ennemi. Et la crainte d'être accusé d'une telle trahison approfondit le sentiment de désaccord entre les partis américains dominants. Pourtant, il existe une catégorie relativement importante bien que sous-représentée, de personnes mécontentes de l'impasse actuelle, mais qui ne veulent pas céder à la simplification excessive et à la polarisation grandissante illustrée par les partisans du Tea Party. C'est eux qui doivent être poussés à exiger que notre discours politique dépasse la polarisation du désaccord profond pour retourner à la tradition de la délibération par le débat public. Certaines des stratégies dont j'ai débattu ici, si elles sont habilement réalisées, pourraient représenter des moyens d'atteindre ce but. Au moins peuventelles fournir un point de départ.

\section{BIBLIOGRAPHIE}

Brockriede, W. 1975. « Where is argument? », Argumentation and Advocacy, 9, 179-182

Dallek, R. 1998. Flawed giant: Lyndon Johnson and his times, 1961-1973 (New York : Oxford University Press)

Ehninger, D. 1958. « Debating as critical deliberation », Southern Communication Journal, 24, 22- 30

Fogelin, R. J. 1985. « The logic of deep disagreements ", Informal Logic, 7, 1-8

Heidlebaugh, N.J. 2001. Judgment, rhetoric, and the problem of incommensurability: Recalling practical wisdom (Columbia : University of South Carolina Press)

Johnstone, H. W., Jr. 1959. Philosophy and argument (University Park : Pennsylvania State University Press)

MacIntyre, A. 1984. After virtue 2d ed. (Notre Dame, IN : University of Notre Dame Press)

Perelman, Ch. \& Olbrechts-Tyteca, L. 1992 [1969]. The New Rhetoric: A Treatise on Argumentation (Notre Dame, IN : University of Notre Dame Press) (édit. française 1958. Traité de l'Argumentation, Ed. de l'Université de Bruxelles)

Schmitt, M. 2010. «Boring politics, please » The American Prospect, 21, 6- 10 
Walton, D. 1998. Ad hominem arguments (Tuscaloosa : University of Alabama Press)

Willard, C.A. 1983. Argumentation and The Social Grounds of Knowledge (Tuscaloosa : University of Alabama Press)

Zarefsky, D. 1990. Lincoln, Douglas, and Slavery: In the crucible of public debate (Chicago : University of Chicago Press)

Zarefsky, D. 2005. Argumentation: The Study of Effective Reasoning (Chantilly, VA : The Teaching Company) [audio and video]

\section{NOTES}

1. Le «Tea Party » est un mouvement politique qui a émergé au début de la présidence d'Obama, composé d'éléments de l'extrême-droite du Parti Républicain, et dont le nom fait référence à la Boston Tea Party de 1773, au cours de laquelle des colons, costumés en Amérindiens, jetèrent par-dessus bord la cargaison de thé de trois navires anglais. Le mouvement s'oppose au système de taxation gouvernemental actuel qu'il trouve excessif, et à l'ingérence du gouvernement central.

2. Rappel : subsomption est un terme de logique, qui se réfère à l'action de penser le particulier sous le général - source: CNRTL (Centre National de Ressources Textuelles et lexicales du CNRS) en ligne (note de la traductrice).

3. Projet de loi portant à la fois sur plusieurs sujets (note de la traductrice).

4. Le compromis de 1850 est un ensemble de projets de lois, passées en septembre 1850, dans le but de désamorcer le conflit entre les États esclavagistes du sud et les États libres du nord sur le statut des territoires acquis pendant la guerre contre le Mexique (1846-1848). Il visait à maintenir l'équilibre au Sénat, où chaque Etat est représenté par deux membres, entre États esclavagistes et non-esclavagistes. Un premier compromis, dit « du Missouri » avait fixé en 1820 une frontière entre États du Sud et États du Nord, au 36,30 de latitude. Aux termes du compromis de 1850, la Californie, bien que située au sud de ce parallèle, est autorisée à intégrer l'Union en tant qu'Etat libre, en échange de quoi les États du Nord s'engagent à restituer à ceux du Sud tout esclave fugitif. De même l'Utah et le Nouveau-Mexique pourraient en principe décider par la suite d'admettre ou d'abolir l'esclavage, bien qu'ils soient situés au nord du parallèle (note de la traductrice).

5. La question de l'extension de l'esclavage aux territoires qui ne faisaient pas encore partie de l'Union avait déjà fait l'objet de nombreux compromis législatifs fragiles entre le Sud esclavagiste et le Nord abolitionniste, notamment en 1821 (« compromis du Missouri »), et en 1850 (voir plus haut note 4). En 1854 cependant, à l'instigation du sénateur démocrate de l'Illinois, Stephen A. Douglas, la loi sur le Kansas-Nebraska, remettant aux habitants des territoires situés au nord du $36^{\mathrm{e}}$ parallèle le soin de fixer leur statut d'États libres ou esclavagistes lors de leur entrée dans l'Union, en vertu du principe de la «souveraineté populaire ", remit la question sur le devant de la scène. Elle donna naissance quelque mois plus tard au nouveau Parti Républicain, dont Lincoln fut l'un des leaders, formé en grande partie pour empêcher l'extension de l'esclavage aux territoires de l'ouest. Décidant de se présenter à l'élection sénatoriale de 1858 contre le sénateur Douglas, Lincoln lui proposa une série de sept débats publics dans différentes villes de l'illinois, débats qui constitueront un épisode exemplaire de la démocratie américaine (Douglas l'emportera cependant par 54 voix contre 46 pour Lincoln). 


\section{RÉSUMÉS}

Le désaccord profond est une situation dans laquelle le conflit est tellement fondamental qu'il ne semble exister à aucun niveau d'accord sous-jacent partagé. Il est généralement admis qu'aucun débat productif n'est possible dans de pareils cas. Tout argument avancé par l'une des parties pourra être contesté par l'autre dans un processus de régression potentiellement infini, car à aucun moment les interlocuteurs ne sont obligés d'accepter un quelconque point de vue en vertu de leurs prises de positions antérieures. Surmonter un désaccord profond exige de dépasser l'impasse sur laquelle achoppe le débat, en envisageant la controverse sous un jour différent. Cet article identifie quatre couples de stratégies rhétoriques permettant de réaménager le désaccord et de transformer le débat. Il présente également deux études de cas pour illustrer ces stratégies : dans l'une, on parvient à dépasser le désaccord, alors que l'autre se solde par un échec.

Deep disagreement is a situation in which a conflict is so fundamental that there appears to be no underlying shared agreement by the arguers at any level. It is generally held that in such a case productive argument is not possible. Any claim the one party makes can be challenged by the other party in a potentially infinite regress, because there is no moment at which the interlocutor, by virtue of his or her prior commitments, is obligated to accept any standpoint. Overcoming deep disagreement requires transcending the impasse in the argument, seeing the controversy in a different light. This essay identifies four pairs of strategies that involve rhetorical moves to reset the disagreement and reshape the argument. In addition, two case studies are presented to illustrate these strategies, one a case of successful transcendence and the other a case of failure.

INDEX

Keywords : abortion controversy, circumstantial ad hominem, deep disagreement, framing, polarization, transcendent argument

Mots-clés : ad hominem circonstanciel, argument transcendant, cadrage, désaccord profond, polarisation, polémique sur l'avortement

\section{AUTEURS}

\section{DAVID ZAREFSKY}

Université Northwestern (USA) 